\title{
More RNAs for oocytes
}

from Pamela Hamlyn

Although Xenopus laevis oocytes are interesting in themselves as a means of studying gene action, their more familiar role is that of an assay system for the translation of injected messenger RNAs. They have many advantages for this purpose, not least their ready availability-several thousand mature oocytes from one adult female. (Compare this with scanning the shelves of health food shops for just the right wheat germ.) Injected messenger RNA is translated with a high efficiency, globin mRNA being translated at nearly the same rate as in intact reticulocytes. Because of this very high efficiency only minute amounts of mRNA are required to produce detectable amounts of protein product and consequently mRNA activity can be detected even when crude RNA fractions containing very little mRNA are injected into oocytes.

van der Donk made use of this property in some experiments that are reported in this issue of Nature, $256,674 ; 1975)$. He is interested in the mechanism by which the style of Petunia hybrida is able to recognise and to differentiate between its own pollen and that of another plant. (Pollen tubes only grow after crosspollination.) Total RNA extracted from self-pollinated styles stimulated

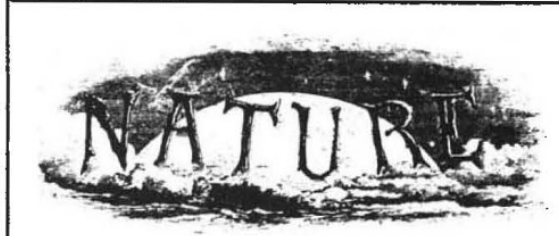

\section{A hundred years ago}

AMONGST the objects which have been recently added to the galleries of the Paris Industrial Exhibition of Geography, and are attracting public notice, we may mention a collection of French birds exhibited by M. Bouvier, the collection of apes from the Gaboon, by the Marquis de Compiègne, and a number of antediluvian fossils from the Mentone Caves. The skeletons of two children which had been buried together are in a splendid state of preservation, exhibiting admirably the characteristics of prehistoric cave-life. These two young people were buried in the home of their parents, very probably because it was the only means of defending their bones against the teeth of ferocious hyænas and other large carnivorous animals which were disputing with man the empire of the future Gaul.

from Nature, 12, 358; August 26, 1875. protein synthesis (by about three times) when injected into $X$. laevis oocytes. A fractionation of the RNA according to molecular weight showed that it was the RNA between $5 \mathrm{~S}$ and18S that gave the biggest stimulation. But stimulation of protein synthesis is completely inadequate as proof that the injected RNA is being translated. Stimulation of endogenous protein synthesis cannot be ruled out as an explanation unless the protein for which the injected messenger codes is shown to be synthesised in the oocytes. The endogenous protein synthesis in the oocyte is higher than in some cell-free systems. In spite of this disadvantage van der Donk was able to demonstrate the appearance of several new protein bands on gel electrophoresis of proteins synthesised in oocytes injected with plant RNA. Nonetheless it could be argued that these bands were oocyte protein whose de novo synthesis was stimulated by the plant RNA. To identify them as plant proteins van der Donk has attempted to demonstrate their function in the inhibition of pollen tube growth. Protein can be isolated from styles after selfpollination which, when transferred to a style that has been cross-pollinated, will inhibit the growth of pollen tubes in that style. Therefore a solution of proteins synthesised in oocytes in the presence and absence of RNA isolated from self-pollinated styles was applied to styles that had been cross-pollinated. Only the proteins synthesised in the presence of the plant RNA were able to inhibit the growth of the pollen tube. Van der Donk takes this as a clear demonstration that at least part of the proteins translated from plant RNA are those involved in the incompatibility reaction (the inhibition of pollen tube growth), and that by partially isolating the RNA coding for these proteins a further step has been made in the elucidation of the intriguing mechanism by which a plant distinguishes between self and non-self.

Gurdon and his colleagues have demonstrated the very long life-up to 10 days-of globin mRNA after injection into oocytes (Gurdon, Lingrel and Marbaix, J. molec. Biol., 80, 539; 1973). Allende and coworkers reasoned that this must be because oocytes contained a very low level of ribonuclease activity or else the RNA was protected in some way from degradation (Allende, Allende and Firtel, Cell, 2, 189; 1974). To distinguish between these two possibilities they injected a variety of radioactive natural and synthetic RNAs into oocytes and followed their degradation with time. They were able to show that some RNAs were degraded and others were not, and concluded that the oocytes contained enough ribonuclease activity to degrade injected RNAs so that those remaining must be protected in some way. They next focused their attention on yeast tRNA-one of the species that survived undegraded in oocytes even after $20 \mathrm{~h}$. In this issue of Nature they report their demonstration that oocytes are capable of aminoacylating yeast tRNAs injected into cells even at final concentrations which were much greater than that of their endogenous tRNA content (page 675). They showed that oocytes can be injected with tRNA for phenylalanine to a final concentration in the cell 500 times the normal concentrationacylation taking place so that there is 150 times as much Phe-tRNA as normal. The authors plan to study the effects of this imbalance on the mechanism of protein synthesis.

\section{Nuclear spectroscopy}

from P. E. Hodgson

IN a nuclear transfer reaction angular momentum is conserved vectorially, so that the spin of the final state of the residual nucleus $\mathbf{J}_{\mathrm{R}}=\mathbf{J}_{\mathrm{T}}+\mathbf{j}$, where $J_{\mathrm{T}}$ is the spin of the ground state of the initial (or target) nucleus and $j$ the angular momentum of the transferred nucleon. This $j$ is itself composed of its orbital and spin angular momenta, $\mathbf{j}=$ $1+\frac{1}{2}$. The angular distribution of the outgoing particle, for example that of a proton in a (d,p) reaction, is usually characteristic of the orbital angular momentum transfer $l$, and thus $l$ can be determined. Since $J_{\mathrm{T}}$ is known, this suffices to set limits on $J_{\mathrm{R}}$ and to determine it in some special cases.

For example, if $J_{\mathrm{T}}=0, \mathbf{J}_{\mathrm{R}}=\mathbf{j}=\mathbf{1}+\frac{1}{2}$, so that $J_{\mathrm{R}}=l \pm \frac{1}{2}$. So if $l=2$, the final state is $d_{3 / 2}$ or $d_{5 / 2}$. This ambiguity may be resolved either by rather difficult measurements of the polarisation of the outgoing particle, or by careful examination of some special features of the differential cross sections (Nature, 250, 464; 1974; 253, 501; 1975).

The situation is more complicated if $J_{\mathrm{T}}$ is greater than zero, for then several values of $j$ may be possible in reactions to states of the same $J_{\mathrm{R}}$. Thus if $J_{\mathrm{T}}=\frac{1}{2}$, $J_{\mathrm{R}}=1$, and $l=1$, then $j$ can be $\frac{1}{2}$ or $3 / 2$. It is an interesting problem to determine $j$, for if this can be done we can learn more about the reaction and also use such reactions to determine $J_{R}$ in cases where it is not known. Furthermore, $j$ has to be known in order to apply the $j$-dependent sum rules to determine nuclear spectroscopic factors (Nature, 249, 695; 1974).

Some years ago Kocher and Haeberli (Phys. Rev. Lett., 23, 315; 1969) showed that the vector analysing power in one-nucleon transfer reactions is 
sensitive to the value of $j$ and thus provides a good way of determining it. They obtained the vector analysing power from the left-right asymmetry of the protons emitted from a reaction initiated by a polarised beam of particles. The $j=\frac{1^{-}}{2}$ reactions ${ }^{32} \mathrm{Cr}(\mathrm{d}, \mathrm{p})^{53} \mathrm{Cr}$ $(Q=5.17 \mathrm{MeV})$ and ${ }^{54} \mathrm{Fe}(\mathrm{d}, \mathrm{p})^{55} \mathrm{Fe}$ $(Q=6.60 \mathrm{MeV})$ at $10 \mathrm{MeV}$ had vector analysing powers quite different from the $j=3 / 2^{-}$reactions ${ }^{52} \mathrm{Cr}(\mathrm{d}, \mathrm{p}){ }^{53} \mathrm{Cr}$ $(Q=5.73 \mathrm{MeV})$ and ${ }^{54} \mathrm{Fe}(\mathrm{d}, \mathrm{p}){ }^{55} \mathrm{Fe}(Q=$ $7.01 \mathrm{MeV})$ at $10 \mathrm{MeV}$. In the mixed- $j$ reaction ${ }^{53} \mathrm{Cr}(\mathrm{d}, \mathrm{p}){ }^{54} \mathrm{Cr} \quad(Q=6.71 \mathrm{MeV})$ at $10 \mathrm{MeV}$, both these values of $j$ were possible, and the vector analysing power closely followed a curve obtained by adding together in definite proportions the vector analysing powers found in the other two reactions. This proportion gave the relative contributions of the $j=\frac{1}{2}$ and $j=3 / 2$ components to the reaction. The technique of finding the mixing parameter in mixed- $j$ transitions has the special advantage of being independent of detailed theoretical calculations, but is somewhat laborious to apply in practice.

A new method of finding the $j$ value has recently been proposed by Dohan and Summers-Gill (Nuclear Phys., A241, 61; 1975), and applied to the ${ }^{69} \mathrm{Gd}(\mathrm{d}, \mathrm{p}){ }^{70} \mathrm{Gd}$ and ${ }^{71} \mathrm{Gd}(\mathrm{d}, \mathrm{t})^{70} \mathrm{Gd}$ reactions to the same state of ${ }^{70} \mathrm{Gd}$. It depends on the fact that, on the simple shell model, the $2 p_{1}: 2$ neutron state is empty in ${ }^{69} \mathrm{Gd}$, half-full (containing one neutron) in ${ }^{70} \mathrm{Gd}$ and full in ${ }^{71} \mathrm{Gd}$. Thus the $(\mathrm{d}, \mathrm{p})$ and $(\mathrm{d}, \mathrm{t})$ reactions on ${ }^{69} \mathrm{Gd}$ and ${ }^{71} \mathrm{Gd}$ respectively go equally well, and thus have very similar spectroscopic factors. The $2 \mathrm{p}_{3 / 2}$ state, on the other hand, is full for all these nuclei, so the $(\mathrm{d}, \mathrm{p})$ reaction on ${ }^{69} \mathrm{Gd}$ is forbidden, as there is no room for another neutron, while the $(\mathrm{d}, \mathrm{t})$ reaction on ${ }^{71} \mathrm{Gd}$ takes place easily as there are plenty of $2 p_{3 ; 2}$ neutrons to be removed. This picture is expected to be somewhat blurred as the simple shell model is not followed exactly, but nevertheless we expect to find very similar amplitudes for $2 \mathrm{p}_{1 / 2}$ transfer and very different amplitudes for $2 \mathrm{p}_{3: 2}$ transfer in the two reactions.

In practice these expectations are remarkably well fulfilled. The transitions to the lower states up to about $1 \mathrm{MeV}$ have almost the same amplitudes for the two types of reaction while those above $1 \mathrm{MeV}$ have very small amplitudes for the $(d, p)$ reaction and large amplitudes for the $(d, t)$ reaction. Thus we can with some confidence assign the first group to $2 \mathrm{p}_{1 / 2}$ transfer and the second group to $2 \mathrm{p}_{3 / 2}$ transfer. This is a very simple and direct way of determining the $j$ value of the transferred particle, and is applicable to reactions where the subshell corresponding to one $j$ value is filling more rapidly than the other. It will be interesting to see how far it can be extended.

In heavy ion reactions the situation is more complicated since the transferred particle can have an angular momentum greater than zero in the projectile. Thus the neutron in the deuteron has $l=0$, whereas the proton in ${ }^{7} \mathrm{Li}$ that is transferred by the $\left({ }^{7} \mathrm{Li},{ }^{6} \mathrm{He}\right)$ reaction has $l=1, j=3 / 2$ as it comes from an $\mathrm{p}_{3 / 2}$ orbit in ${ }^{7} \mathrm{Li}$ (Nature, 253, 501; 1975).

\section{Sticky actin}

from Dennis Bray

MuSCLE biochemistry is not usually the place to look for the bizarre; but what else could one call an association between muscle actin and an enzyme that digests DNA? The story is a curious one. As long ago as 1943, it was fcund that pigeon thymus contains a protein that inhibits deoxyribonuclease I-the abundant endonuclease of pancreas. This activity was found in other tissues but little else was learnt about its biochemistry until the late 60 s when Lindberg - at the Karolinska Institute, Stockholm-began a careful study. He found that the inhibitor had a molecular weight close to 42,000 ; that it could be purified and even crystallised by conventional procedures; and that it formed a tight $1: 1$ complex with the nuclease. Among its notable characteristics, at least in retrospect, was its abundance in tissue $(5-10 \%$ of the protein in a soluble extract), and its tendency to form aggregates of high molecular weight.

Then last year, at Cold Spring Harbor, New York, Iazarides and others interested in cell motility heard at first-hand of the DNase inhibitor. They also had a protein with molecular weight close to 42,000 which was strangely abundant in cells: only they called it actin. The amino acid compositions of the two proteins were encouragingly similar and the unlikely possibility was put to the test. Sure enough, the fingerprints of the two are the same, they cross react immunolog.cally, and analytical-grade muscle actin sticks so tightly to DNase I that denaturing agents are needed to part them (Lazarides and Lindberg, Proc. natn. Acad. Sci. U.S.A., 71, 4742; 1974).

The interaction is unquestionably real but why does it occur? It is unlikely to be accidental because the binding is so tight. It may have some purpose, but it links two cellular processes that are normally considered to be independent. The puzzle is compounded by indications that actin forms other unexpected associations. Before the work mentioned above, Laki and Muszbek (Biochim. biophys. Acta.,
371, 519; 1974), had shown that actin in its filamentous form binds strongly to fibrin--the major structural protein of blood clots. And a recent paper examines the long known association between glycolytic enzymes, such as aldolase and triose phosphate dehydrogenase, and filamentous actin (Clark and Masters, Biochim. biophys. Acta., 381, 37; 1975). Still only in abstract form are claims that an interaction exists between actin and spectrin, the major protein of red blood cell ghosts (Tilney, J. Cell Biol., 63, 349a; 1974); and another report that actin might be identical to the $\gamma$-subunit of dogfish muscle phosphorylase (Fischer et al., Hoppe-Seylers Z. Sür Physiol. Chem., 356, 381; 1975)

If we consider the proteins that associate with thin filaments in muscle -troponin, tropomyosin, myosin and actinin-then we have ten or so that all bind to actin. The list might well be longer, for any club that includes both deoxyribonuclease and myosin must surely have a wide membership. It begins to look as though we are in the presence of a Phenomenon; but what it might mean is hard to say. Either actin is generally sticky and able to adhere to proteins it does not normally encounter, or many proteins have cvolved binding sites that hitch them to this abundant and phylogenetically stable component of the cell.

\section{Evolution of $E$. coli chromosome}

\section{from Millicent Masters}

Ir has been possible to observe the intact chromosome of about half-adozen species of bacteria. In each species the DNA was found to be a closed circle. The circular chromosome of the best studied species of bacterium, Escherichia coli, contains enough DNA to code for about 3,000 average-sized polypeptide chains. Specific functions have been assigned to about 500 of these genes and the well developed genetics of $E$. coli has permitted their positions on the chromosome to be determined. Genes specifying proteins with related functions such as isoenzymes catalysing the same reaction or groups of enzymes involved in sequential metabolic conversions may be either close together or distant on the circular chromosome. Some related genes are close together because they are transcribed together (that is, they form an operon). Other related genes however are close together without sharing such a common control mechanism. Still other related genes, such as the five genes concerned with pyrimidine synthesis or seven of the 11 genes responsible for arginine biosyn- 